\title{
Intra-platelet Serotonin as a Biomarker in HCC Recurrence: When Time Matters
}

\author{
Bibek Aryal ${ }^{1}$, Munekazu Yamakuchi ${ }^{2}$, Teruto Hashiguchi ${ }^{\circledR}$, Yutaka Imoto ${ }^{1}$ \\ 1. Cardiovascular and Gastroenterological Surgery, Graduate School of Medical and Dental Sciences, Kagoshima University 890-8520, Kagoshima, Japan \\ 2. Department of Laboratory and Vascular Medicine, Graduate School of Medical and Dental Sciences, Kagoshima University, Kagoshima 890-8520, Japan \\ $\triangle$ Corresponding author: Teruto Hashiguchi, Department of Laboratory and Vascular Medicine, Graduate School of Medical and Dental Sciences, Kagoshima \\ University, 8-35-1, Sakuragaoka, Kagoshima 890-8520, Japan. Email: terutoha@m3.kufm.kagoshima-u.ac.jp Telephone: (+81)-99-275-5437; Fax: (+81)-99-275- \\ 5437; \\ (C) Ivyspring International Publisher. This is an open access article distributed under the terms of the Creative Commons Attribution (CC BY-NC) license \\ (https://creativecommons.org/licenses/by-nc/4.0/). See http://ivyspring.com/terms for full terms and conditions.
}

Received: 2018.10.15; Accepted: 2019.04.15; Published: 2019.05.26

Related article: J Cancer 2018; 9(20):3713-3714. doi:10.7150/jca.27497

We thank Yang et al. for their attention to our work and concomitant description of their findings. In our investigation, we demonstrated a post-resection depletion of intra-platelet (IP) serotonin (5-HT) concentrations in patients with hepatocellular carcinoma (HCC) [1]. We observed an exhaustive pattern of post-resection platelet kinetics in patients with early HCC recurrence. While the post-resection serum and IP 5-HT levels were significantly depleted in patients with HCC recurrence, the preoperative concentration, although observed a similar tendency, this did not reach statistical significance. Interestingly, Yang et al. observed that the high serum 5-HT, high IP 5-HT and high IP 5-HT per platelet were all associated with poor overall and recurrence-free survival [2]. In accordance with it, Xia et al. reported that a preoperative elevated 5-HT was associated with advanced tumor node metastasis and poor recurrence-free survival and overall survival [3]. Similarly, in another study by Padickakudy et al., higher levels of preoperative IP 5-HT were found to be associated with improved postoperative liver regeneration, and an increase in early tumor recurrence suggesting a bivalent property of IP 5-HT in liver regeneration and post-resection recurrence [4].

Collectively, all the above-mentioned studies monitored the kinetics of IP 5-HT in post-resection cancer recurrence. Although there appears to be only a subtle difference between the designs of the studies, our study has some critical disparities. Our data explicitly refers to a post-resection time point (four weeks after liver resection), while the other studies focused on preoperative time points.

Platelets exhibit a variety of qualitative abnormalities in patients with cancer (before or after surgery) [5,6]. These deviations comprise reduced, elevated or spontaneous platelet aggregation, and hypersensitivity to various platelet agonists. A state of oxidative stress was reported in resting blood platelets obtained from cancer patients [7]. Platelet proteome harbors differentially expressed proteins associated with tumors that were found normalized after tumor resection [8]. Likewise, partial hepatictomy also highly influences platelet functions. A hypercoagulable state elicited as a result of liver resection combined with the active promitogenic effect of platelets in liver regeneration stimulates platelet activation [9]. In this context, identifying an optimum time-point of blood sampling is crucial to aptly translate the prognostic or predictive value of post-resection platelet kinetics in cancer patients. Although our study on post-resection IP kinetics at four-weeks post-resection has not assessed the absolute advantage of this time point, it has technically minimized the biases from the confounding factors including the presence of tumor or immediate post-resection related stresses.

Another issue that needs to be mentioned is the method of platelet preparation. Mussbacher et al. have stressed on a significant heterogeneity among anticoagulants used to prevent unwanted platelet activation [10]. Platelets are highly sensitive to changes in the microenvironment, they are prone to in vitro activation during platelet-preparation. 
Optimized sample preparation is crucial to investigate platelet granule release and preventing artifacts due to in vitro platelet activation. There are also some discrepancies in the analysis of IP 5-HT between different studies; in the study by Shu et al., IP 5-HT was calculated by subtracting the plasma 5-HT level from the serum 5-HT level [3] whereas, in our investigations, although not as optimized as mentioned by Mussbacher et al., we precisely isolated platelets and monitored the IP growth factors in the platelet extracts [1]. Our results showed that along with IP 5-HT exhaustion, there was also exhaustion of platelet counts, which is in accordance with the phenomenon observed by Shehta et al. [11]. Along with IP 5-HT, we also observed similar propensity with other platelet-related growth factors including angiopoetin-1 and platelet-derived growth factor. The potential pathophysiological significance of postresection platelet exhaustion was not explored in our previous study. Our published (and ongoing) studies indicate that a highly stochastic phenomenon along with the differential secretion of IP growth factor is orchestrated in patients with post-resection HCC recurrence.

In considerations with all these factors, we suggest that the discrepancies observed in these (apparently) similar studies should better be readdressed with full-consideration to the platelets' disease-specific, site-specific and stage-specific response [12]. Taking everything into account, not only IP 5-HT but also all platelet-based growth factors stability should be studied under different conditions to identify the most efficient protocol in-regards to the timing, collection, and handling.

\section{Acknowledgements}

Dr. Bibek Aryal is a fellow of Japan Society for the Promotion of Science (JSPS). The work mentioned in this manuscript was supported by the program of Grants-in-Aid for Scientific Research from JSPS, KAKENHI Grant numbers: JP 16F16420, JP 18F16420 and JP $16 \mathrm{H} 05229$.

\section{Competing Interests}

The authors have declared that no competing interest exists.

\section{References}

1. Aryal B, Shimizu T, Kadono J, et al. Post-Resection Exhaustion of Intra-Platelet Serotonin: Also an Indicator of Early Hepatocellular Carcinoma Recurrence? Journal of Cancer. 2017;8(19):3984-91.

2. Yang Q, Liu S, Deng C, Shu B, et al. Preoperative Serum and Intra-platelet Serotonin in Prognosis: Useful or Useless? J Cancer 2018; 9(20):3713-3714.

3. Shu B, Wang S, Deng $\mathrm{Y}$, et al. Intra-platelet serotonin in prognosis of tumorigenesis: Friend or foe? Journal of hepatology. 2018;68(6):1333-4.

4. Padickakudy R, Pereyra D, Offensperger F, et al. Bivalent role of intra-platelet serotonin in liver regeneration and tumor recurrence in humans. Journal of hepatology. 2017;67(6):1243-52.
5. Goad KE, Gralnick HR. Coagulation disorders in cancer. Hematology/ Oncology Clinics of North America 1996;10:457-483.

6. Kedzierska M, Czernek U, Szydłowska-Pazera K, et al. The changes of blood platelet activation in breast cancer patients before surgery, after surgery, and in various phases of the chemotherapy. Platelets. 2013;24(6):462-8.

7. Kedzierska M, Olas B, Wachowicz B, et al. The nitrative and oxidative stress in blood platelets isolated from breast cancer patients: the protectory action of aronia melanocarpa extract. Platelets. 2010;21(7):541-8.

8. Sabrkhany S, Kuijpers MJE, Knol JC, et al. Exploration of the platelet proteome in patients with early-stage cancer. Journal of Proteomics. 2018 Apr 15;177:65-74.

9. Meyer J, Lejmi E, Fontana P, et al. A focus on the role of platelets in liver regeneration: Do platelet-endothelial cell interactions initiate the regenerative process? Journal of Hepatology. 2015 Nov;63(5):1263-71.

10. Mussbacher M, Schrottmaier WC, Salzmann M, et al. Optimized plasma preparation is essential to monitor platelet-stored molecules in humans. PLoS One 2017;12:e0188921.

11. Shehta A, Han HS, Ahn S, et al. Post-resection recurrence of hepatocellular carcinoma in cirrhotic patients: Is thrombocytopenia a risk factor for recurrence? Surgical oncology. 2016; 25: 364-9.

12. Chauhan A, Adams DH, Watson SP, et al. Platelets: No longer bystanders in liver disease. Hepatology. 2016 Nov;64(5):1774-1784. 